\title{
Eletropolimerização de azul de metileno sobre carbono vítreo e estabilidade do filme de poli(azul de metileno)
}

The electropolymerization of methylene blue on glassy carbon and the stability of poly(methylene blue) film

\author{
W. A. Braun, R. C. Kussler, M. Bagatini, M. Hilgemann* \\ Centro de Ciênicas Extas e Tecnológicas, Centro Universitário UNIVATES, CEP 95900-000, Lajeado-RS, Brasil
}

*mauricio.hilgemann@univates.br

(Recebido em 16 de maio de 2016; aceito em 27 de julho de 2016)

\begin{abstract}
Os polímeros condutores (PCs) têm permeado muitos campos da pesquisa eletroquímica. O trabalho tem como objetivo estudar diferentes condições para a polimerização eletroquímica do azul de metileno (MB) sobre carbono vítreo e testar a estabilidade dos filmes poliméricos (PMB) formados, a fim de que possam vir a ser usados como biomembranas. $\mathrm{O}$ estudo da eletropolimerização do MB foi feito através da técnica de voltametria cíclica, na faixa de potencial de $-0,40 \mathrm{a}+1,20 \mathrm{~V}$, a uma velocidade de varredura de $50 \mathrm{mV} \mathrm{s}^{-}$ 1. Os parâmetros estudados para a eletropolimerização foram o uso de diferentes soluções eletrolíticas, o potencial inicial aplicado e um pós-tratamento, que consistia em manter o filme polimérico na solução eletrolítica contendo o monômero MB por $24 \mathrm{~h}$ a $4{ }^{\circ} \mathrm{C}$. Para a avaliação da estabilidade dos filmes formados, voltamogramas cíclicos foram obtidos na faixa de potencial de $-0,40$ a $+0,50 \mathrm{~V}$, com uma velocidade de varredura de $50 \mathrm{mV} \mathrm{s}^{-1}$, em intervalos de 5 minutos entre cada leitura, utilizando uma solução de tampão acetato $(\mathrm{pH} 5,0)$ e $\mathrm{NaNO}_{3} 0,1 \mathrm{~mol} \mathrm{~L}^{-1}$. Na maioria dos cenários testados, o sinal da corrente do PMB decai com o passar do tempo. No entanto, dentre as várias condições testadas, a que apresentou melhores resultados envolve deixar o eletrodo mergulhado na solução eletrolítica, o que proporciona que o monômero ainda presente no filme possa ser lixiviado de volta à solução, e garante um sinal estável com o passar do tempo.

Palavras-chave: azul de metileno, eletropolimerização, polímeros condutores
\end{abstract}

Conducting polymers (CP) have permeated many electrochemical research fields. The work aims to study different conditions for the electrochemical polymerization of methylene blue (MB) on glassy carbon and test the stability of the polymer films (PMB) formed, so that they might be used as biomembranes. The study of the electropolymerization of MB was done by cyclic voltammetry, in the potential range of -0.40 to $+1.20 \mathrm{~V}$ at a scan rate of $50 \mathrm{mV} \mathrm{s}^{-1}$. The parameters studied for electropolymerization were the use of different electrolytic solutions, the initial potential applied and a post-treatment, consisting of keeping the polymeric film in its electrolyte solution for $24 \mathrm{~h}$ at $4{ }^{\circ} \mathrm{C}$. To evaluate the stability of the formed films, cyclic voltammograms were obtained in the potential range of -0.40 to $+0.50 \mathrm{~V}$, with a scan rate of $50 \mathrm{mV}$ $\mathrm{s}^{-1}$, at intervals of 5 minutes between each reading, using an acetate buffer solution $(\mathrm{pH} 5.0)$ and $0.1 \mathrm{~mol} \mathrm{~L}^{-1}$ $\mathrm{NaNO}_{3}$. For most of the tested scenarios, the current signal of PMB decays over time. However, among various tested conditions, the best results were obtained when the electrode was left to age in electrolyte overnight. This allowed unpolymerised monomer retained within the film to escape and led to a more stable response over time.

Keywords: methylene blue, electrolymerization, conducting polymers

\section{INTRODUÇÃO}

$\mathrm{O}$ alto potencial de aplicações de polímeros condutores (PCs) em sensores químicos e biológicos é uma das principais razões para uma intensa investigação e desenvolvimento destes materiais [1]. Durante uma reação, os PCs podem apresentar mudanças em suas propriedades, tais como volume, cor, carga armazenada, porosidade e molhabilidade, de maneira que filmes de PCs também podem mimetizar propriedades similares a órgãos em funcionamento [2]. Por razões práticas, polímeros condutores que podem ser preparados a partir de compostos baratos, como anilina, pirrol, flavinas e seus derivados, através de um processo relativamente simples de polimerização eletroquímica, atraem grande interesse. Entretanto, polímeros redox também são aplicados em casos especiais, como em biossensores ou aparelhos eletroquímicos [2-7]. O uso de 
PCs na tecnologia de sensores envolve utilizá-los como uma modificação da superfície de um eletrodo para aumentar sua sensibilidade, transmitir seletividade, diminuir interferências e providenciar uma matriz de suporte para as moléculas do sensor [1]. As várias propostas de aplicação necessitam que o polímero condutor apresente diferentes propriedades. Portanto, a síntese de um novo tipo de polímero condutor é muito significativa. Eletrodos com a superfície modificada baseados na eletropolimerização de fenazinas, fenotiazinas e fenoxazinas já foram reportados na literatura [8]. A eletropolimerização é uma das técnicas mais simples e úteis para se obter filmes poliméricos. Dentre os PCs, o poli(azul de metileno) (PMB) tem sido amplamente estudado por sua atividade eletrocatalítica na presença de biomoléculas e diferentes compostos inorgânicos. Karyakin et al. [9] foram os primeiros a reportar a polimerização eletroquímica do azul de metileno (MB). O filme polimérico foi usado como biomaterial devido a sua atividade bioeletroquímica. A principal vantagem da eletropolimerização do $\mathrm{MB}$ deve-se à simples obtenção do polímero, sintetizado a partir de uma solução aquosa do monômero [10].

Assim, o trabalho teve como objetivo estudar diferentes condições para a polimerização eletroquímica do azul de metileno sobre carbono vítreo e testar a estabilidade dos filmes poliméricos formados, a fim de que possam vir a ser usados como biomembranas ou biossensores em trabalhos futuros.

\section{MATERIAL E MÉTODOS}

\subsection{Reagentes e soluções}

Todos os reagentes utilizados neste trabalho foram de grau analítico, e as soluções foram preparadas com água ultrapura.

A concentração utilizada do monômero MB (Quimex) em todas as soluções foi de $0,76 \mathrm{mmol}$ $\mathrm{L}^{-1}$. Os eletrólitos suportes utilizados para a eletropolimerização do $\mathrm{MB}$ foram $\mathrm{NaCl}, \mathrm{Na}_{2} \mathrm{SO}_{4}$ ou $\mathrm{NaNO}_{3}$, todos a uma concentração de $0,1 \mathrm{~mol} \mathrm{~L}^{-1}$, e contendo $0,02 \mathrm{~mol} \mathrm{~L}^{-1}$ de tetraborato de sódio $(\mathrm{pH}=9,2)[8]$.

\subsection{Equipamentos}

Todas as medidas eletroquímicas foram realizadas num sistema de 3 eletrodos, em que um fio de platina era o contra-eletrodo, o eletrodo de $\mathrm{Ag} / \mathrm{AgCl}$ em $\mathrm{KCl} 3 \mathrm{M}$ era usado como referência e o eletrodo de carbono vítreo (GCE) foi usado como eletrodo de trabalho. As medidas foram realizadas em um potenciostato/galvanostato PGSTAT128N (AUTOLAB). Todos os experimentos foram realizados à temperatura ambiente e utilizou-se uma gaiola de Faraday para minimizar possíveis interferências elétricas.

\subsection{Preparação do eletrodo de PMB modificado}

Antes da modificação da superfície do eletrodo de trabalho, este foi mecanicamente polido com alumina $0,5 \mu \mathrm{m}$. Após, o eletrodo foi lavado com etanol para remover partículas residuais que poderiam estar aderidas à superfície. Finalmente, o eletrodo foi sonicado em solução água/etanol (1:1) por 5 min, e então lavado com etanol e água.

A modificação da superfície do GCE foi executada pela técnica de voltametria cíclica nas soluções de eletrólitos suportes descritas acima, contendo o monômero a uma concentração de $0,76 \mathrm{mmol} \mathrm{L}^{-1}$. Os voltamogramas cíclicos foram obtidos na faixa de $-0,4 \mathrm{~V}$ a $+1,2 \mathrm{~V}$ versus $\mathrm{Ag} / \mathrm{AgCl}$ por 30 ciclos, a uma velocidade de varredura de $50 \mathrm{mV} \mathrm{s}^{-1}$.

Os parâmetros estudados para a eletropolimerização foram o uso de diferentes soluções eletrolíticas, o potencial inicial aplicado $(-0,4$ ou $+1,2 \mathrm{~V})$ e um pós-tratamento, que consistia em manter o filme de PMB por 24 h na solução eletrolítica contendo o monômero MB a $4{ }^{\circ} \mathrm{C}$.

\subsection{Avaliação da estabilidade do filme de PMB}

Após a modificação do eletrodo de trabalho, um voltamograma cíclico foi obtido na faixa de potencial de $-0,40$ a $+0,50 \mathrm{~V}$, a uma velocidade de varredura de $50 \mathrm{mV} \mathrm{s}^{-1}$, utilizando uma solução de tampão acetato $(\mathrm{pH} 5,0)$ e $\mathrm{NaNO}_{3} 0,1 \mathrm{~mol} \mathrm{~L}^{-1}$. Então, o eletrodo era deixado em 
repouso por 5 minutos na solução e um novo voltamograma era registrado após este período. Esta última etapa foi repetida mais 4 vezes, totalizando uma diferença de 30 minutos entre o primeiro e o último voltamograma.

A avaliação da estabilidade dos filmes de PMB formados foi feita pela medida do decréscimo da carga $\left(Q_{\downarrow} / Q_{0}\right)$ sobre o pico de oxidação do filme polimerizado sobre o eletrodo no intervalo de 30 minutos. Após este procedimento, a superfície do eletrodo era regenerada pelo polimento mecânico, lavagem e sonicagem.

Todas as medidas foram realizadas em triplicata.

\section{RESULTADOS E DISCUSSÃO}

A eletropolimerização do MB atinge taxas máximas de crescimento em meio básico, conforme já relatado por vários autores $[4,8,10-12]$, inclusive sendo mostrado que, para o caso específico do $\mathrm{MB}$, apenas uma pequena quantidade de polímero é obtida a partir de soluções ácidas $[9,13]$. Portanto, a polimerização eletroquímica do MB foi realizada conforme Karyakin et al. [8], em pH 9,2 , aplicando-se 30 ciclos e variando-se os eletrólitos suportes $\left(\mathrm{Cl}^{-}, \mathrm{SO}_{4}{ }^{2-}\right.$ e $\left.\mathrm{NO}_{3}{ }^{-}\right)$, todos a uma concentração de $0,1 \mathrm{~mol} \mathrm{~L}^{-1}$. Os eletrólitos suportes $\mathrm{Cl}^{-}$e $\mathrm{SO}_{4}{ }^{2-}$ não apresentaram bons resultados para a eletropolimerização do MB uma vez que não houve a formação do filme polimérico. Consequentemente, a sua utilização foi descartada. Assim, optou-se pela utilização de $\mathrm{NaNO}_{3}$ como eletrólito suporte para a formação do PMB sobre carbono vítreo.

O perfil voltamétrico do eletrodo modificado com PMB (Figura 1) apresenta dois picos redox e um terceiro pico em que ocorre apenas um processo oxidativo. $O$ primeiro pico redox, em $-0,12$ $\mathrm{V}$, está relacionado com a oxidação do monômero MB. Conforme a eletropolimerização prossegue, este pico redox decresce, e um novo conjunto de picos aparece nos voltamogramas cíclicos entre $-0,2 \mathrm{~V}$ e $+0,2 \mathrm{~V}$, indicando uma atividade polimérica do tipo redox que tende a potenciais mais positivos em comparação com o monômero. Investigações eletroquímicas e espectroeletroquímicas já confirmaram que este novo par redox que surge no curso da deposição pode ser atribuído ao polímero resultante [14]. O pico de oxidação observado próximo a 1,2 V está relacionado com a formação de espécies radicalares catiônicas, uma vez que o monômero MB é carregado positivamente, e são necessárias para o processo de eletropolimerização $[8,12]$. $\mathrm{O}$ pico redox relacionado com a formação do polímero apresenta um comportamento quasireversível, como pode ser visto na Figura 1. Com o aumento no número de ciclos, o perfil voltamétrico torna-se mais irreversível uma vez que a distância entre os picos de oxidação e redução aumenta com o número de ciclos, sem, no entanto, observar-se um aumento significativo na corrente de pico. Este mesmo comportamento foi observado por Marinho et al. [12]. O potencial inicial aplicado $(-0,4$ ou $+1,2 \mathrm{~V})$ não teve qualquer influência na formação do filme polimérico formado. 


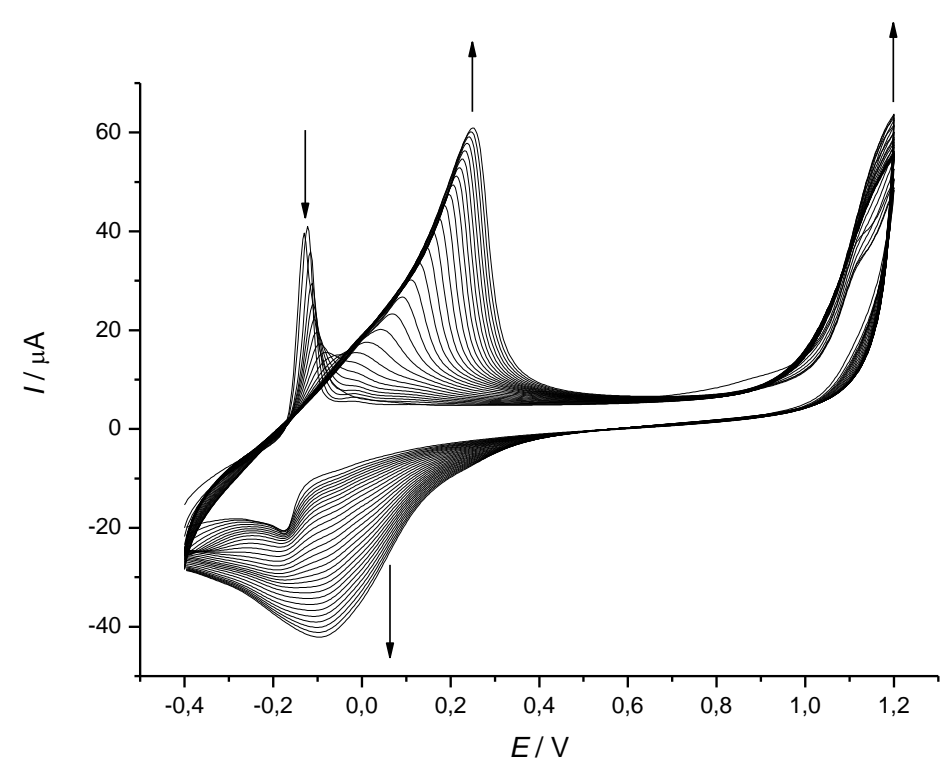

Figura 1: Perfil voltamétrico da eletropolimerização do $\mathrm{MB} ;[\mathrm{MB}]=0,76 \mathrm{mmol} \mathrm{L}^{-1}$ em $\mathrm{Na}_{2} \mathrm{~B}_{4} \mathrm{O}_{7} 0,02 \mathrm{~mol}$ $L^{-1}$ e $\mathrm{NaNO}_{3} 0,1 \mathrm{~mol} \mathrm{~L}^{-1}(\mathrm{pH} 9,2) ; 30$ ciclos entre $-0,4$ e $+1,2 \mathrm{Va} 50 \mathrm{mV} \mathrm{s}^{-1}$.

O parâmetro que influenciou diretamente na estabilidade do filme formado foi a realização de um pós-tratamento, que consistia em manter o filme de PMB por $24 \mathrm{~h}$ na solução eletrolítica contendo o monômero MB a $4{ }^{\circ} \mathrm{C}$. Na Figura 2 é possível ver a importância deste tratamento para a estabilidade do filme de PMB, já que os valores de $Q$ decaem significativamente com o tempo quando da não realização do mesmo. Após a eletropolimerização, o eletrodo modificado é deixado em repouso na solução eletrolítica para permitir que o monômero não-polimerizado retido no filme possa ser lixiviado para obter-se uma resposta mais estável [4, 12].

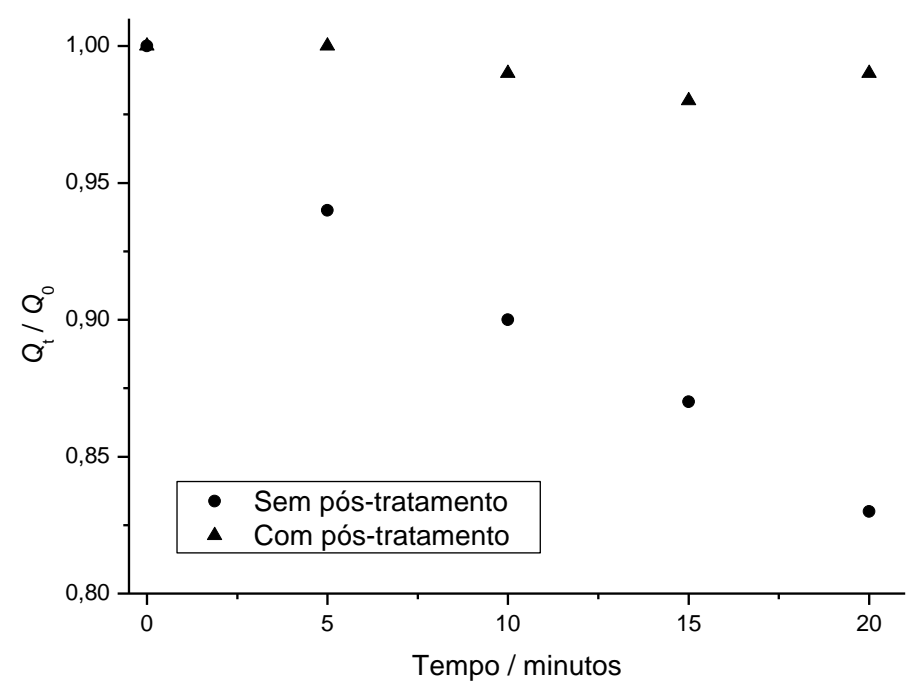

Figura 2: Valores de $Q_{t} / Q_{0}$ em função do tempo, obtidos com e sem pós-tratamento. 
De fato, o filme polimérico formado na superfície do eletrodo é bastante estável em função do tempo. A Figura 3 apresenta os voltamogramas cíclicos do eletrodo modificado com PMB obtidos em uma solução de $\mathrm{NaNO}_{3} 0,1 \mathrm{~mol} \mathrm{~L}{ }^{-1}$ e tampão acetato $0,05 \mathrm{~mol} \mathrm{~L}^{-1}(\mathrm{pH}=5,0)$, de $-0,4$ $\mathrm{V} \mathrm{a}+0,5 \mathrm{~V}$, a uma velocidade de varredura de $50 \mathrm{mV} \mathrm{s}^{-1}$, obtidos dentro de um intervalo de 30 minutos para se estudar a estabilidade do filme formado através do tempo. Cada voltamograma foi obtido em intervalos de 5 minutos entre as medidas. Como se pode perceber, o filme polimérico aderido ao eletrodo se mostra bastante estável com o tempo, sendo que a diferença dos valores de carga entre o primeiro e o último voltamograma varia menos de $6 \%$.

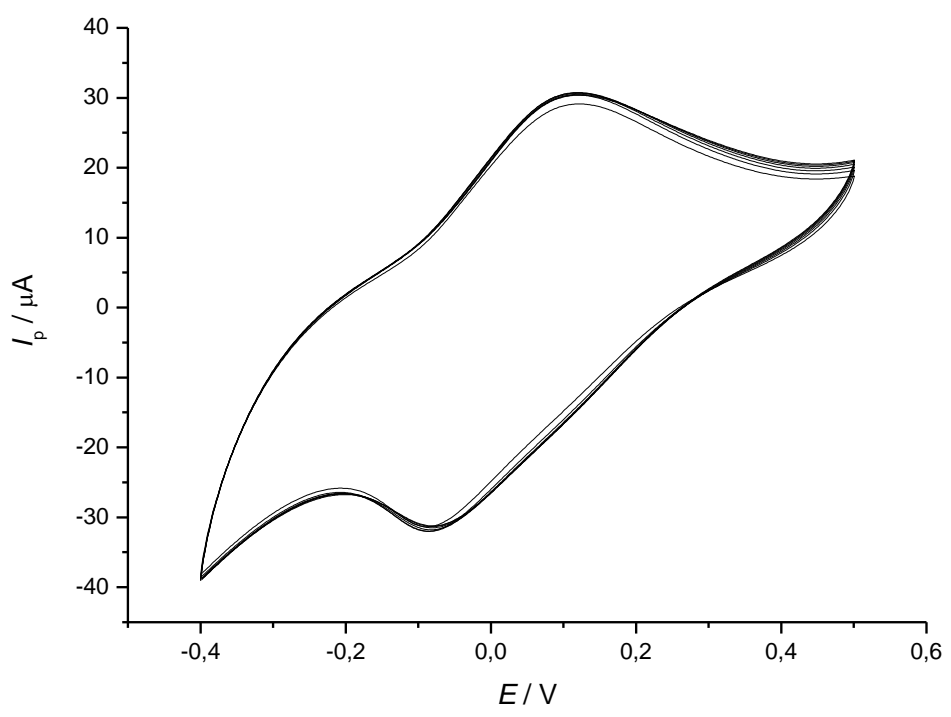

Figura 3: Voltamogramas cíclicos registrados com o eletrodo modificado com $P M B$ em 0,1 mol L $L^{-1}$ de $\mathrm{NaNO}_{3}$ e 0,05 mol $\mathrm{L}^{-1}$ de tampão acetato $(\mathrm{pH}$ 5,0). Cada voltamograma foi obtido em intervalos de 5 minutos entre cada medida, a uma velocidade de varredura de $50 \mathrm{mV} \mathrm{s}^{-1}$.

A cobertura superficial do filme de PMB na superfície do eletrodo de GC pode ser estimada pela concentração superficial $(\Gamma)$ do polímero. O valor de $\Gamma$ foi calculado pela carga $(Q)$ associada com o processo de polimerização, de acordo com $[12,15]$ :

$$
\Gamma=Q /(n \cdot F \cdot A)
$$

em que $A$ é a área geométrica do eletrodo de $\mathrm{GC}\left(0,070 \mathrm{~cm}^{2}\right), n$ é o número de elétrons envolvidos no processo redox $(n=2)$ e $F$ é a constante de Faraday $\left(96.485 \mathrm{C} \mathrm{mol}^{-1}\right)$.

$\mathrm{O}$ valor de $Q$ foi calculado pela integração do pico de oxidação do PMB obtido com uma velocidade de varredura de $50 \mathrm{mV} \mathrm{s}^{-1}(Q=15.0 \mu \mathrm{C} \pm 1.33)$. Portanto, o valor de $\Gamma$ é $1,11 \times 10^{-9}$ mol $\mathrm{cm}^{-2}$. O valor de $\Gamma$ obtido revela uma boa cobertura superficial do filme de PMB na superfície do eletrodo de GC. Consequentemente, é possível assumir boa estabilidade física e química durante as medidas eletroquímicas.

\section{CONCLUSÃO}

Na maioria dos cenários testados, o sinal da corrente do PMB decai com o passar do tempo. No entanto, obteve-se uma resposta estável quando, após a eletropolimerização, manteve-se o eletrodo mergulhado na solução eletrolítica contendo o monômero $\mathrm{MB}$ por $24 \mathrm{~h}$ a $4{ }^{\circ} \mathrm{C}$, o que proporciona que o monômero ainda presente no filme possa ser lixiviado de volta à solução, e garante um sinal estável com o passar do tempo. Assim, o filme de PMB mostrou-se viável como 
sensor eletroquímico, pois é possível formá-lo com relativa facilidade e se mostra bastante estável com o passar do tempo.

\section{AGRADECIMENTOS}

A CNPq e Fapergs pelos auxílios e bolsas concedidos.

\section{REFERÊNCIAS BIBLIOGRÁFICAS}

1. Inzelt G. Conducting Polymers: A New Era in Electrochemistry. Berlin Heidelberg: Springer; 2008. 282 p.

2. Otero TF, Martinez JG, Arias-Pardilla J. Biomimetic electrochemistry from conducting polymers. A review. Artificial muscles, smart membranes, smart drug delivery and computer/neuron interfaces. Electrochim. Acta. 2012 Dec;84(1):112-28, doi: 10.1016/j.electacta.2012.03.097.

3. Silber A, Hampp, N, Schuhmann W. Poly(methylene blue)-modified thickfilm gold electrodes for the electrocatalytic oxidation of NADH and their application in glucose biosensors. Biosens. Bioelectron. 1996 Mar;11(3):215-23, doi: 10.1016/0956-5663(96)88408-9.

4. Brett, CMA, Inzelt, G, Kertesz, V. Poly(methylene blue) modified electrode sensor for haemoglobin. Anal. Chim. Acta. 1999 Apr;385(1-3):119-23, doi:10.1016/S0003-2670(98)00808-3.

5. Mahmoudian MR, Alias Y, Basirun WJ, Golsheikh AM, Jamali-Sheini. Synthesis of polypyrrole coated manganese nanowires and their application in hydrogen peroxide detection. Mater. Chem. Phys. 2013 Aug;141(1):298-303; doi:10.1016/j.matchemphys.2013.05.014.

6. Gualandi I, Guadagnini L, Zappoli S, Tonelli D. A Polypyrrole Based Sensor for the Electrochemical Detection of OH Radicals. Electroanalysis. 2014 Jun;26(7):1544-50, doi: 10.1002/elan.201400054.

7. Li Y, Li W, Zhou H, Wang F, Chen Y,Wang Y, Yu C. A facile method for the sensing of antioxidants based on the redox transformation of polyaniline. Sens. Actuators B Chem. 2015 Mar;208(1):30-5, doi:10.1016/j.snb.2014.11.018.

8. Karyakin AA, Karyakina EE, Schmidt H-L. Electropolymerized Azines: A New Group of Electroactive Polymers. Electroanalysis. 1999 May;11(3):149-55, doi: 10.1002/(SICI)15214109(199903)11:3<149::AID-ELAN149>3.0.CO;2-G.

9. Karyakin AA, Strakhova AK, Karyakina EE, Varfolomeyev SD, Yatsimirsky AK. The electrochemical polymerization of methylene blue and bioelectrochemical activity of the resulting film. Bioelectrochem. Bioenerg. 1993 Sep;32(1):35-43, doi:10.1016/0302-4598(93)80018-P.

10. Kaplan IH, Dagci K, Alanyahoglu M. Nucleation and Growth Mechanism of Electropolymerization of Methylene Blue: The Effect of Preparation Potential on Poly(methylene blue) Structure. Electroanalysis. 2010 Nov;22(22):2694-2701, doi: 10.1002/elan.201000304.

11. Liu J, Mu S. The electrochemical polymerization of methylene blue and properties of polymethylene blue. Synth. Met. 1999 Dec;107(3):159-65, doi:10.1016/S0379-6779(99)00146-0.

12. Marinho MIC, Cabral MF, Mazo LH. Is the poly (methylene blue)-modified glassy carbon electrode an adequate electrode for the simple detection of thiols and amino acid-based molecules? J. Electroanal. Chem. 2012 Oct;685(1)8-14, doi:10.1016/j.jelechem.2012.08.023.

13. Cosnier S, Karyakin AA. Electropolymerization: Concepts, Materials and Applications. Weinheim (Germany): Wiley-VCH Verlag GmbH \& Co. KGaA; 2010. Chapter 5, Electropolymerized Azines: a New Group of Electroactive Polymers; p. 93-110, doi: 10.1002/9783527630592.ch5.

14. Schlereth DD, Karyakin AA. Electropolymerization of phenothiazine, phenoxazine and phenazine derivatives: Characterization of the polymers by UV-visible difference spectroelectrochemistry and Fourier transform IR spectroscopy. J. Electroanal. Chem. 1995 Oct;395(1-2):221-32, doi:10.1016/00220728(95)04127-A.

15. Yogeswaran U, Chen S-M. Multi-walled carbon nanotubes with poly(methylene blue) composite film for the enhancement and separation of electroanalytical responses of catecholamine and ascorbic acid. Sens. Actuators B Chem. 2008 Mar;130(2):739-49, doi:10.1016/j.snb.2007.10.040. 